\title{
Research Progress in Acoustical Application to Petroleum Logging and Seismic Exploration
}

\author{
Lin $\mathrm{Fa}^{*}{ }^{* 1}$, Lei Wang ${ }^{1}$, Yuan Zhao ${ }^{2}$, Lin Liu ${ }^{1}$, Yajuan Zheng ${ }^{1}$, Nan Zhao ${ }^{2}$, Meishan Zhao ${ }^{2}$ and Guohui $\mathrm{Li}^{1}$ \\ ${ }^{1}$ School of Electronic Engineering, Xi'an University of Post and Telecommunications, Xi'an, Shaanxi 710121, China \\ ${ }^{2}$ The James Frank Institute and Department of Chemistry, The University of Chicago, Chicago, Illinois 60637, USA
}

\begin{abstract}
This paper is concerned with an improved network model in acoustical application to petroleum logging and seismic exploration. Utilizing acoustic-electric analogue, we report in this paper a newly developed acoustic-logging network model. Important relationships amongst various physical factors are established, i.e. driving-voltage signal, electric-acoustic conversion of source-transducer, acoustic-electric conversion of receiver-transducer, the physical and geometrical properties of propagation media, as well as the measured logging signal. Technically, a driving-voltage convolution with electric-acoustic impulse response is used to substitute for some traditionally assumed acoustic-source functions on acoustic logging, e.g. Tsang wavelet, Ricker wavelet, Gaussian impulse wavelet, etc. With an improved understanding of the anisotropic effects on reflection/refraction between two different anisotropic rock slabs, the new network model can be used to determine the various properties of signal propagation in acoustic-logging, including propagation speed, phase factor, signal amplitude, and frequency information. In turn, it provides input for analysis of amplitude variations with offset (AVO). Corresponding to the improved network model, with available logging and seismic exploration data, a new algorithm for analysis of amplitude variation has been developed to explore new oil reservoirs or gas fields.
\end{abstract}

Keywords: Acoustic-logging, transducer, reflection/refraction, inversion, oil reservoir, seismic exploration, seismic signal and data analysis.

\section{INTRODUCTION}

Reflection and refraction of plane waves at the interface between two media are amongst the most fundamental processes in wave propagation. They form the basis for seismic forward modeling and seismic amplitude variations with offset (AVO) data analysis. The properties of plane waves at the interface of different media have been investigated extensively and reported elsewhere, such as the quality detection of concrete structures, as reported by Larose etc [1]. These studies were aimed primarily at achieving an improved understanding of the physical properties and geometric structure of the propagation media.

Neglecting the impacts of electric-acoustic and acousticelectric conversions of transducer on acoustic-logging signals, traditional acoustic logging methods usually adopt some assumed mathematical functions, such as Tsang wavelet, Gaussian pulse wavelet, etc. to describe an acoustic source [2-4]. These approaches have advanced theoretical guidance significantly to many applications. Technically, they simplified physical and mathematical analyses and provided reasonable results in many cases of petroleum

*Address correspondence to this author at the School of Electronic Engineering, Xi'an University of Post and Telecommunications, Xi'an, Shaanxi 710121, China; Tel: 0086-29-88166264;

E-mail: fa_yy@yahoo.com.cn logging and seismic exploration. Nevertheless, it must be noted that this traditional simplification without proper justification can lead to significant error in many practical applications.

The electric-acoustic and acoustic-electric conversions could cause transmission delay and amplitude/frequency variation of a measured signal. It is exactly due to this reason that traditional logging methods are far from perfect in practical work on petroleum logging and seismic exploration. Indeed, for accurate analysis in practical applications of petroleum logging, an improved network model needs to be established. Drawing on analogy between acoustic logging process and signal transmission, we have established a new network model which is more favorable and accurate for analysis of acoustic-logging transmission.

It should be noted that the majority of oilfields in China, as well as in many parts of the world, have entered into the mid- or late-stage of exploitation. It becomes much more challenging to raise the oil and natural gas yield for the existing reservoirs or even to stabilize the production. Therefore, precise methods to discover new oil and gas reservoirs are urgently needed, especially for thin-layer oilgas reservoirs. Building on a regional geological model and applying logging data from several oil-wells in the China region, we report in this paper a newly developed algorithm in constructions of the so called seismic wavelet dictionary. 
It is well known that elastic anisotropy is ubiquitous in Earth's interior [5]. The effects of the rock anisotropy on the reflection coefficient are usually calculated using the measured rock anisotropy parameters, such as these parameters as reported by Thomson [6]. The results of calculations may be used in amplitude variations with offset (AVO) analysis for reflection/refraction coefficients. The reflection coefficients of rock formations inside the earth may be obtained by matching regional seismic data with the constructed seismic wavelet dictionary, i.e. seismic wavelets constructed by using logging data. The amplitude and phase of the refection coefficients may be used to locate the oil-gas reservoirs, e.g. the thin-layer oil-gas reservoirs [7].

\section{ACOUSTIC-LOGGING AND SEISMIC EXPLORATION}

\subsection{Relationship Between Radiated Acoustic-Signal and Driving-Voltage Signal}

Let's consider the relationship between driving-voltage signal and acoustic-signal radiated by the source-transducer, as well as that of acoustic-signal and electric-signal converted from the receiver-transducer.

For the radiated acoustic-signal and driving-voltage signal, let's consider a spherical thin-shell transducer with acoustic-electric and electric-acoustic equivalence. The circuits of the transducer are established, as shown in Fig. (1a, b). For these two equivalent circuits, by solving piezoelectric and particle movement equations, the electricacoustic and acoustic-electric impulse responses can be obtained.

The electric-acoustic and acoustic-electric impulse response functions may be written as [8-10]

$h_{1}(t)=K_{1} e^{-\alpha_{1} t}+K_{2} e^{-\beta_{1} t} \cos \left(\omega_{1} t-\theta_{1}\right)$,

$h_{3}(t)=K_{3} e^{-\alpha_{3} t}+K_{4} \mathrm{e}^{-\beta_{3} t} \cos \left(\omega_{3} t-\theta_{3}\right)$.

In Eqs. (1)-(2), $K_{1}, K_{2}, K_{3}$ and $K_{4}$ are constants; $\alpha_{1}$ and $\beta_{1}$ are the damping coefficients for the direct current and alternate current of $h_{1}(t) ; \alpha_{3}$ and $\beta_{3}$ are those for the direct current and alternate current of $h_{3}(t) ; \theta_{1}$ and $\theta_{3}$ are the phase shifts; $\omega_{1}$ and $\omega_{3}$ are the center frequencies of the source-transducer and receiver-transducer.

The driving-voltage convolution function with the electric-acoustic impulse response from the transducer may be used to substitute for the traditionally assumed acousticsource functions, such as Tsang wavelet, Gaussian pulse wavelet, etc which makes the acoustic logging forward model to be much closer to the actual acoustic logging.

Now, let's construct a system with the following conditions and do a practical calculation for electric-acoustic and acoustic-electric conversion. The transducer is composed of the piezoelectric material PZT-7A [11], the coupling medium around the transducer is the transformer oil, and the output impedance of the driving circuit is taken to be $50 \Omega$. We also use the physical and geometrical parameters of the thin-shell transducer as shown in Table 1. From Eqs. (1)-(2), the calculated electric-acoustic and acoustic-electric conversions in time- and frequency-domains of the transducer are presented in Figs. $(\mathbf{2}, \mathbf{3})$. The various symbol notations used in these figures and throughout the paper are defined in Table 2. A gated sine voltage signal as shown in Fig. (4) is used to excite the source-transducer. Acoustic signal radiated by the source-transducer is as shown in Fig. (5).

(a)

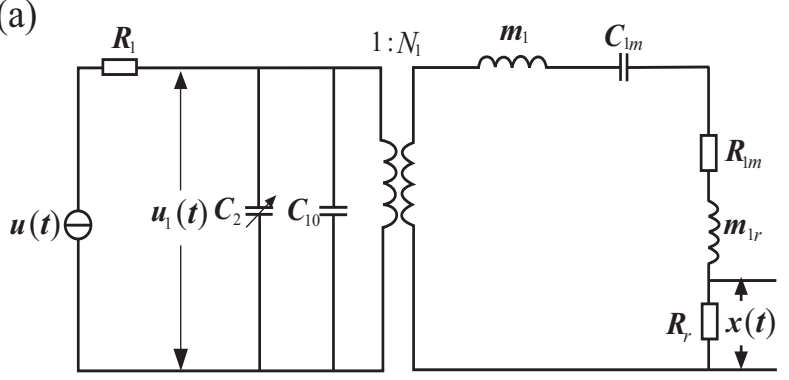

(b)

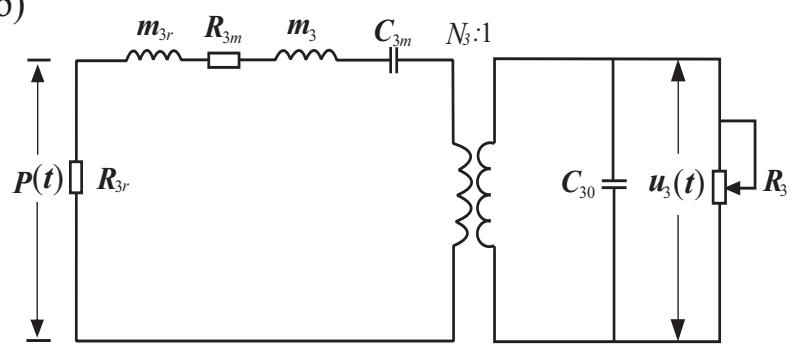

Fig. (1). Two equivalent circuits of the transducers: (a) the sourcetransducer; (b) the receiver-transducer.

\subsection{Acoustic-Logging Transmission Network Model}

Now, let's consider the geometrical configuration of an acoustic logging as shown in Fig. (6). A logging tool is placed in a fluid-filled cylindrical borehole and it is embedded in an infinitely large medium. $T$ and $R$ are the source-transducer and receiver-transducer respectively with a distance $L$ from $T$ to $R$. Electric driving signal excites $T$ to emit acoustic signal which propagates to $R$ via the borehole mud or the formations surrounding the borehole. Then, the acoustic signal is converted into electric signal by $R$ and recorded by the logging tool [12].

It is noted that the transducer impact on amplitude and frequency of a logging signal can be significant, mainly contributed by electric-acoustic and acoustic-electric conversion. Nevertheless, none of the traditional acousticlogging methods has ever put that into consideration. The traditional acoustic-logging models simply neglect the transmission time delay caused by both the electric-acoustic conversion and the acoustic-electric conversion. In our improved model, the actual travel time of an acousticlogging signal in media is obtained from the propagation time measured by a traditional logging device minus all pieces of transmission time delay.

Based on signal transmission theory, a model acousticlogging transmission network (ALTN) may be established to analyze the total acoustic-logging process, shown in Fig. (7). Surpassing traditional acoustic logging models, an ALTN 
Table 1. Physical and Geometrical Parameters of Transducer and Acoustic Impedance of the Coupling Medium: in the Table $\rho$ is the Density of Transducer Material; $d_{31}, \varepsilon_{33}^{T}, s_{12}^{E}$ and $s_{11}^{E}$ are the Piezoelectric Dielectric and Strain Constants Respectively; $Z_{m}$ is the Acoustic-Impedance of Coupling-Medium Around the Transducer; $r_{b}$ and $l_{t}$ are the Average Radius and the Shell Thickness of the Transducer

\begin{tabular}{|c|c|c|c|c|c|c|c|}
\hline $\begin{array}{c}\rho \times 10^{3} \\
\left(\mathrm{~kg} / \mathrm{m}^{3}\right)\end{array}$ & $\begin{array}{c}d_{31} \times 10^{-12} \\
(m / V)\end{array}$ & $\begin{array}{c}\varepsilon_{33}^{T} \times 10^{-9} \\
(F / m)\end{array}$ & $\begin{array}{l}s_{12}^{E} \times 10^{-12} \\
\left(m^{2} / N\right)\end{array}$ & $\begin{array}{l}s_{11}^{E} \times 10^{-12} \\
\left(m^{2} / N\right)\end{array}$ & $\begin{array}{c}Z_{m} \times 10^{6} \\
\left(\mathrm{~kg} / \mathrm{m}^{2} \cdot \mathrm{s}\right)\end{array}$ & $\begin{array}{c}r_{b} \\
(\mathrm{~cm})\end{array}$ & $\begin{array}{c}l_{t} \\
(\mathrm{~cm})\end{array}$ \\
\hline 7.6 & -60 & 3.7613 & -3.2 & 10.7 & 1.2205 & 8 & 0.8 \\
\hline
\end{tabular}

Table 2. Definitions of Symbols Shown in Fig. (1)

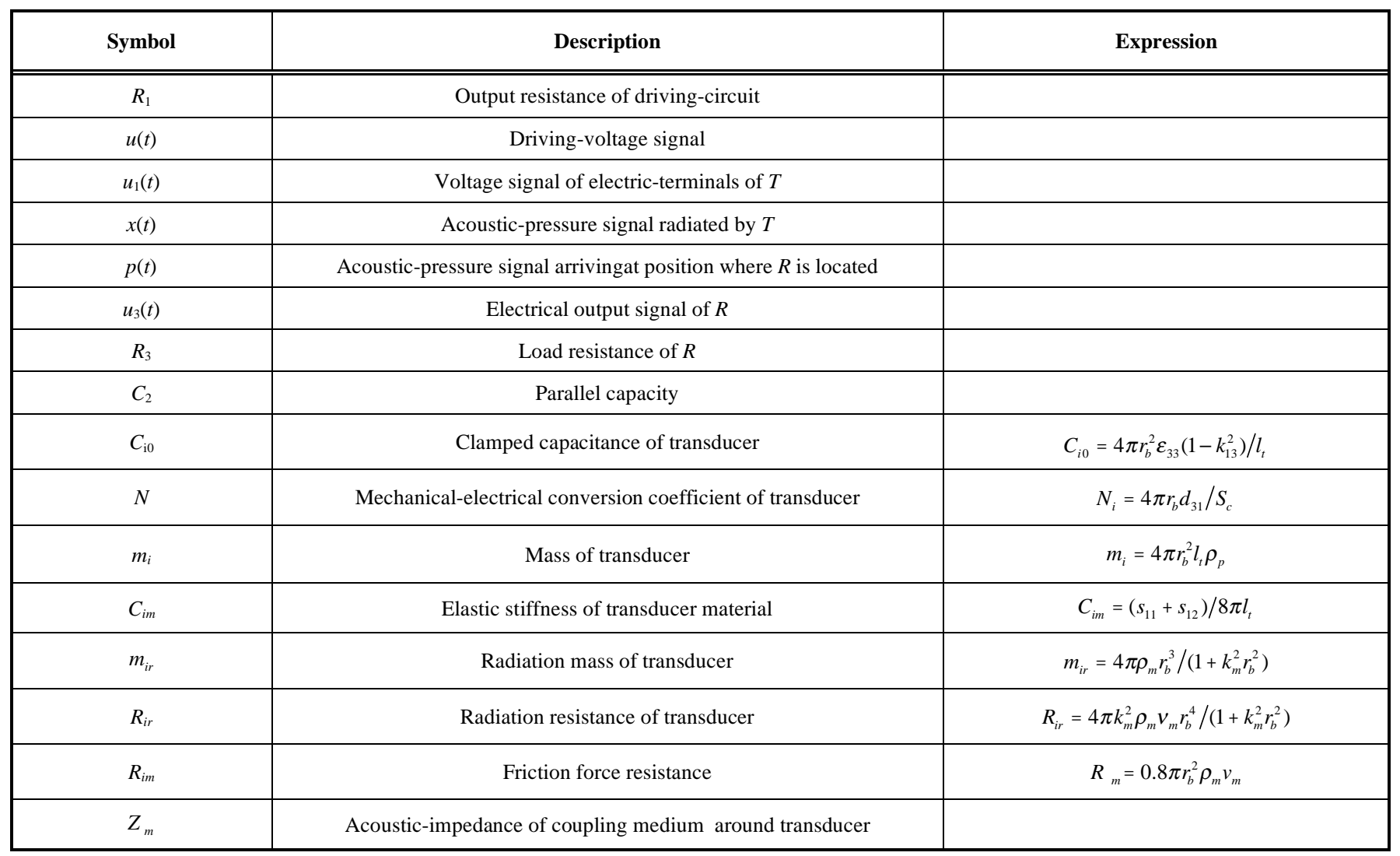

model takes a full consideration on the effect of electricacoustic and acoustic-electric conversion of a logging signal, including transmission time delay caused by the transducers. Therefore, an ALTN model yields more accurate measurement on propagation speed, signal amplitude, and frequency information of logging signals.

The ALTN model in Fig. (7) can be used to describe the geometrical configuration of acoustic-logging as shown in Fig. (6). In this model, the driving-voltage signal $u_{1}(t)$ and the measured logging signal wavelet $u_{3}(t)$ are defined as the input and output respectively. The measured signal $u_{3}(t)$ is a summery contribution from several factors, including electric-acoustic conversion of $T$, the physical and geometrical properties of the propagation media (borehole mud or the formation around borehole), and acoustic-electric conversion of $R$ on the driving-voltage signal $u_{1}(t)$. We may consider $T$ as an electric-acoustic filter, the propagation media as an acoustic filter, and $R$ as an acoustic-electric filter. We also define $x(t)$ as the acoustic signal radiated by $T$, and $p\left(t, z_{0}\right)$ as the acoustic signal wavelet propagating to $R$, via the borehole fluid and the formation near the borehole.

To emphasize the transmission time delay caused by the acoustic-electric conversion of $R$, Tsang wavelet may be used for the acoustic-pressure signal radiated by $T$, which takes the form

$$
x(t)=4 \alpha t e^{-\alpha t} \sin \left(\omega_{0} t\right) H(t)
$$

The parameter $\alpha$ is a damping coefficient and $\omega_{0}$ is the center frequency of the wavelet. 

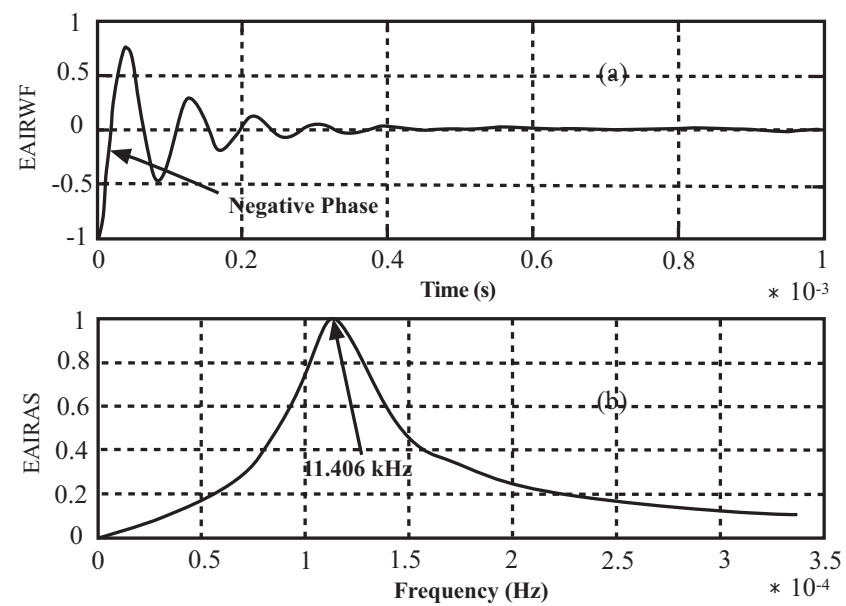

Fig. (2). Electric-acoustic conversion of source-transducer: (a) electric-acoustic impulse response; (b) amplitude spectrum. EAIRWF stands for the magnitude of the electric-acoustic impulse response waveform and EAIRAS is that of the corresponding amplitude spectrum.
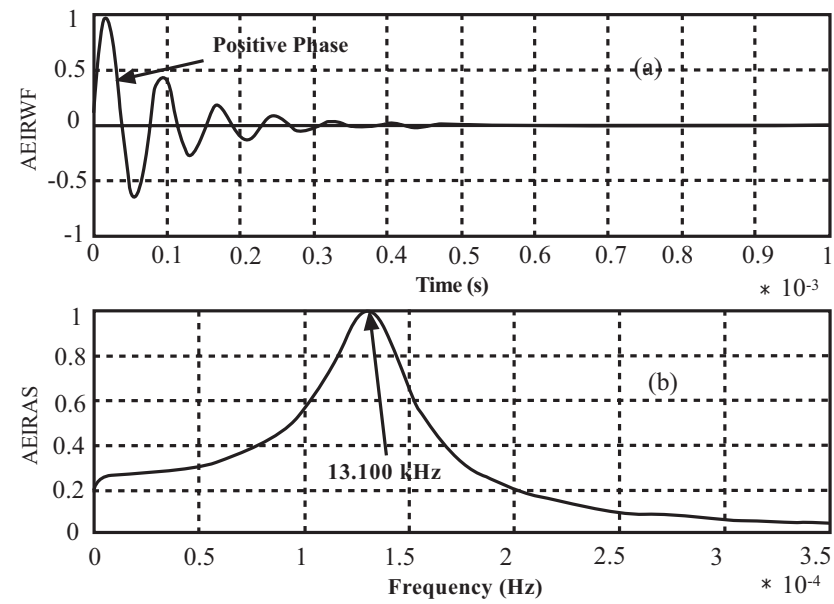

Fig. (3). Acoustic-electric conversion of receiver-transducer: (a) acoustic-electric impulse response; (b) amplitude spectrum. AEIRWF stands for the magnitude of acoustic-electric impulse response waveform and EAIRAS is that of the corresponding amplitude spectrum.
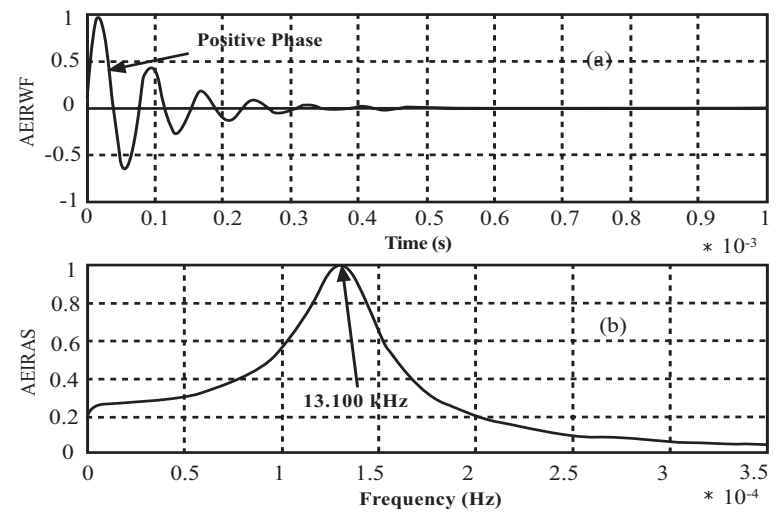

Fig. (4). Driving-voltage signal: (a) the waveform; (b) amplitude spectrum. NEWF stands for the magnitude of the normalized driving-voltage signal waveform and NEAS is that of the corresponding amplitude spectrum; $t$ is the abbreviation of time and $f$ is that of frequency.

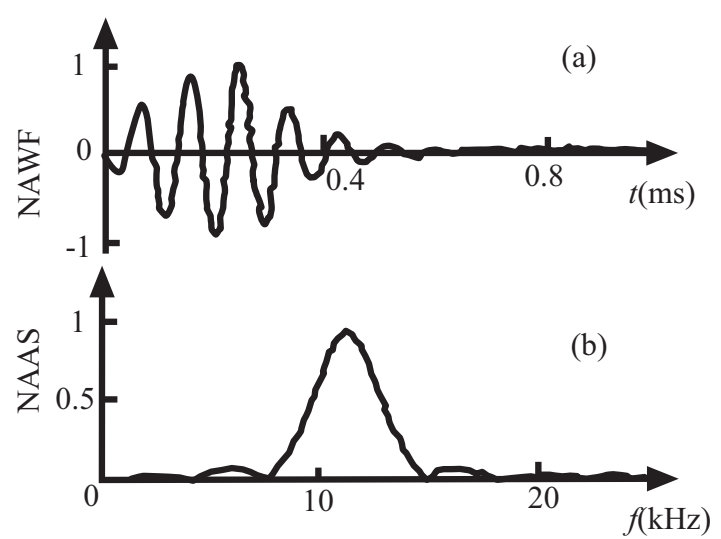

Fig. (5). Radiated acoustic signal: (a) the waveform; (b) amplitude spectrum. NAWF is the waveform radiated by the source and NAAS is the corresponding amplitude spectrum.

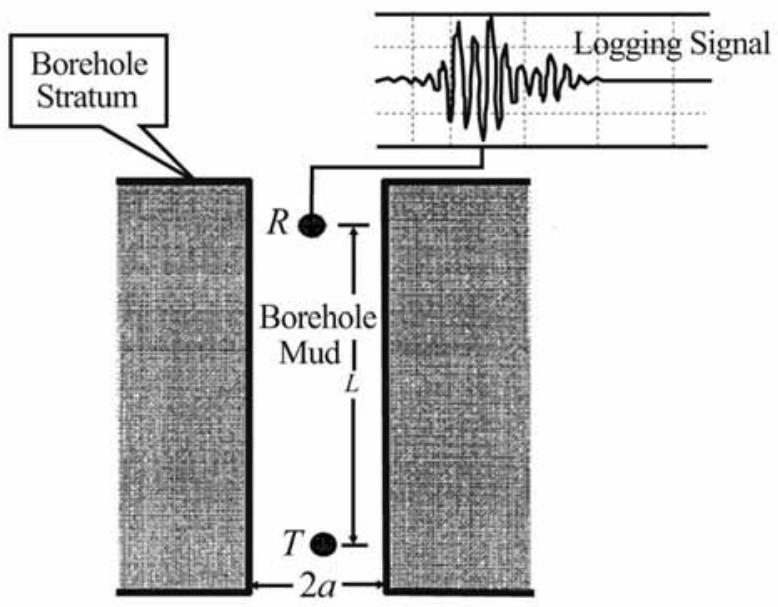

Fig. (6). A schematic plot of the geometrical configuration of an acoustic-logging system: $T$ and $R$ are respectively the sourcetransducer and receiver-transducer with a distance $L$ from $T$ to $R$.

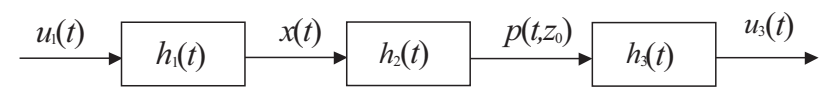

Fig. (7). A model acoustic-logging transmission network (ALTN).

We have performed calculations of wavelet for waveform and amplitude spectrum using parameters listed in Table $\mathbf{3}$ $[12,13]$. For the entries in Table $3, \rho$ is the density of propagation medium, $v_{p}$ is the acoustic speed of $P$-wave, and $v_{s v}$ is the acoustic speed of $S V$-wave in media. The distance " $L$ " from $T$ to $R$ is set to be $1.3224 m$ and the borehole radius " $a$ " is at $12 \mathrm{~cm}$. The Tsang wavelet parameters are $\omega_{0}=2 \pi \times 10^{4} \mathrm{rad} / \mathrm{s}, \quad \alpha=0.6 \omega_{0} / \pi$. The calculated results are shown in Fig. $(\mathbf{8})$. Fig. $(\mathbf{8 a}, \mathbf{b})$ are the normalized Tsang wavelet for waveform and amplitude spectrum, $(\mathbf{c}, \mathbf{d})$ are those of acoustic signal $p\left(t, z_{0}\right)$ reaching at $R$ via the borehole fluid and the formation around the borehole, and $(\mathbf{e}, \mathbf{f})$ are those of electric signal $u_{3}(t)$ converted by $R$.

The calculated results in Fig. (8) show that for the acoustic-logging signal wavelet, the transmission delay caused by the acoustic-electric conversion of $R$ can be up to 
$3.1886 \mu$ s. Compared to the negative head wave amplitude value of $p(t, z)$ in Fig $(\mathbf{8 c})$, the head wave amplitude of $u_{3}(t)$ in Fig. (8e) has initially a relative decline and then changes to a positive value. It shows that if the effect of acousticelectric conversion of $R$ on the logging signal is neglected, the measured value of the speed will produce a much larger error.

Table 3. Physical Parameters of the Borehole Fluid and MCSandstone Around Borehole

\begin{tabular}{|c|c|c|c|}
\hline Medium & $\rho\left(\boldsymbol{k g} / \mathbf{m}^{3}\right)$ & $\boldsymbol{v}_{p}(\boldsymbol{m} / \boldsymbol{s})$ & $\boldsymbol{v}_{\boldsymbol{s}}(\boldsymbol{m} / \mathbf{s})$ \\
\hline \hline Borehole fluid & 1.2 & 1540 & \\
\hline Formation & 2.16 & 5943 & 3200 \\
\hline
\end{tabular}
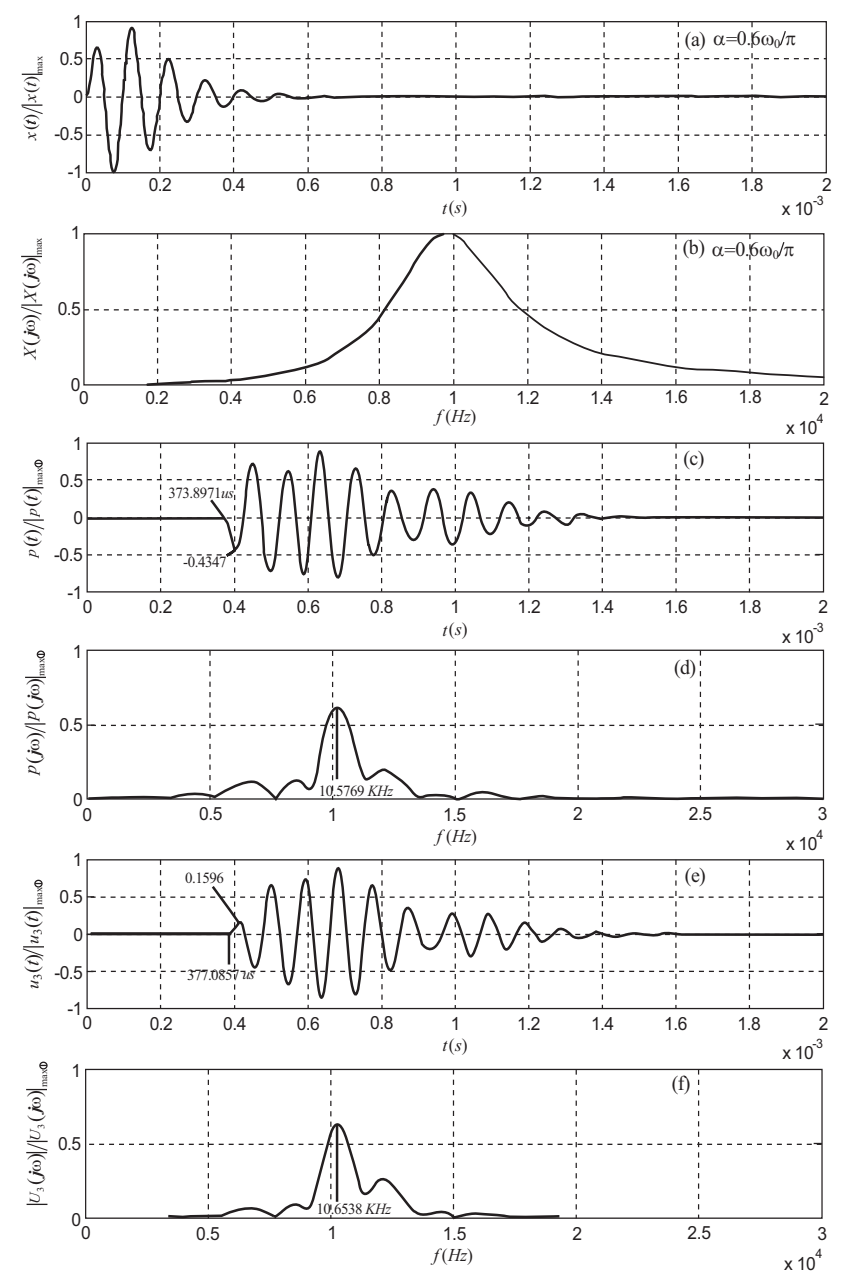

Fig. (8). Acoustic-electric conversion of receiver-transducer on the measured logging signal: (a, b). the normalized Tsang wavelet spectrum for waveform and amplitude; (c, d). those of acoustic signal $p\left(t, z_{0}\right)$ reaching at $R$ via the borehole fluid and the formation around the borehole; $(\mathbf{e}, \mathbf{f})$. those of electric signal $u_{3}(t)$ converted by $R$.

In traditional cement bond quality logging, if the headwave amplitude of acoustic-logging signal is small, the cement bond quality of the cased-well is defined as good; otherwise, the cement bond quality is considered as poor. Now, if the impact of acoustic-electric conversion of $R$ on the head-wave amplitude is not properly considered, the bad cementation quality may be misjudged as a good cementation quality and vice versa [14].

\subsection{Addition and Multiplication ALTNs}

For an array acoustic-source (AAS) in the borehole at the radiation directivity maximum, the excitation time delay $\Delta t$ for two neighbor transmitting elements, i.e. the transducers in ALTN model, is given by $[15,16]$

$\Delta t=d / v$,

where, $d$ is the interval between two neighboring transmitting elements and $v$ is the acoustic velocity of $P$ wave in the formation around the borehole. Numerical analysis has shown that an array acoustic-source ALTN model with a single receiver is reciprocal to that of a single source with an array acoustic-receiver (AAR).

Eq. (4) may be used to adjust the excitation time delay of an array acoustic-source ALTN model with a single receiver. It is easy see that the head-wave amplitude of acousticlogging signal linearly increases with respect to the number of transducer elements in the AAS. Similarly, to adjust the shifting time of acoustic-logging signals received by each receiving elements in ALTN with a single source-transducer, the head-wave amplitude of acoustic-logging signal increases also roughly linearly with respect to the number of transducer elements in the AAR. So, the above mentioned two ALTNs abide approximately by an addition rule and are identified as the addition ALTN.

Assume that we have an ALTN network model with $N$ transducers in AAS and $M$ transducers in AAR. For the ALTN with AAS and AAR, $P$-wave velocity around the borehole is used to adjust the AAS excitation time delay. The acoustic signals emitted by all transmitting elements in AAS propagate around the borehole with the same phase. Then, the propagating signals reach the receiving elements in AAR and change into electric signals due to the acousticelectric conversion of the transducers. Finally, the shifting time of these electric signals converted by each receiving elements may be adjusted by solving Eq. (4). The value of the stacked head-wave amplitude increases approximately as a product of $M$ and $N$. In this case, the ALTN model is identified as a multiplication ALTN.

Now, let's consider a simple example with $N=4$ and $M=$ 4 , i.e. AAS and AAR consist of four transducers each. The interval between two neighboring transducers is set to be 82 $\mathrm{mm}$ and the distance from AAS to AAR is taken as $2.44 \mathrm{~m}$. The calculated acoustic beam directivities for this ALTN are presented in Fig. (9). Clearly, the acoustic-beam steering efficiency of the multiplication ALTN is much higher than that of the addition ALTN. In either case, two equivalent ALTNs, either addition or multiplication, would increase greatly the head wave amplitude for the acoustic-logging signal. 


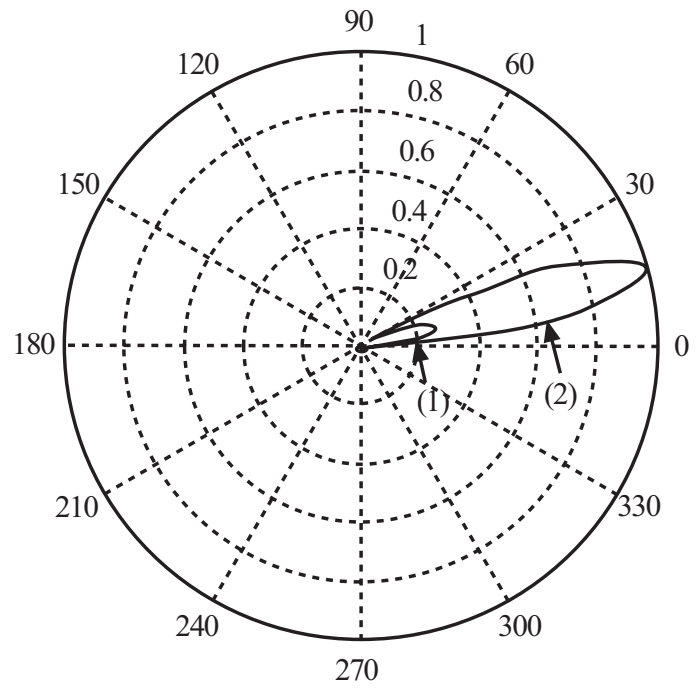

Fig. (9). Acoustic-beam steering directivities of addition and multiplication ALTNs: curve (1) is the directivities of the two addition ALTNs and curve (2) is that of the multiplication ALTN.

\subsection{Acoustic Signal Propagation in Drill-Collar}

Acoustic logging while drilling is mainly used for the acoustic velocity measurement around horizontal wells, deviated wells on land, and cluster wells on offshore.

Conventional logging method can be applied to acousticlogging tool while drilling. Acoustic probes may be used as either the general acoustic-transducers or multiple acoustictransducers. The acquired logging data could be processed by a proper software program which is written on the electric circuit module in the acoustic logging tool while drilling (ALTWD).

The drill-collar is usually made of steel and the outershell of ALTWD is a steel-grooves casing. The ALTWD is placed in drill collar and the outer-shell of ALTWD is scheduled for torsion force created during drilling. Bearing huge torsion force, the drill collar cannot be grooved, or else it will be ruined. As shown in Fig. (10), there are small windows on the drill-collar and on the outer-shell of ALTWD. These windows are located in the vicinity of source transducers and receiver transducers. The acoustic signal radiated by the source-transducer in ALTWD can pass through the pipe layers via the windows, and then reach the formation around the borehole. The acoustic-signal coming from the formation can be collected by receiver-transducers in ALTWD.

One of the technical difficulties with ALTWD is that the propagation speed of $P$-wave in steel is typically about 5900 $\mathrm{m} / \mathrm{s}$. This speed is usually larger than that of low- or intermediate-velocity formation in ALTWD. The propagation path of acoustic signal in drill-collar is actually shorter than that of formation in ALTWD. Without a specific design, the acoustic signal from the drill-collar may reach receiver-transducer unwittingly ahead of the signal from the formation around the borehole.

To make a specific design, we note that scattering takes place when acoustic-signal impinges on an interface between two different media. Applying acoustic-scattering theory and adopting medium acoustic-absorption technology, the outershell of drill-collar may be properly designed with some required specifications. By doing so, the outer-shell ensures that acoustic signal emitted by the source-transducer would pass fully through the windows on outer-shell of ALTWD, as well as on drill collars. Due to the acoustic-scattering and acoustic-absorption of the drill-collar inner-wall ensures that the acoustic signal reaching the drill-collar is scattered and attenuated. This will ensure the acoustic logging signals come from the formation around the borehole, rather than from drill-collar.

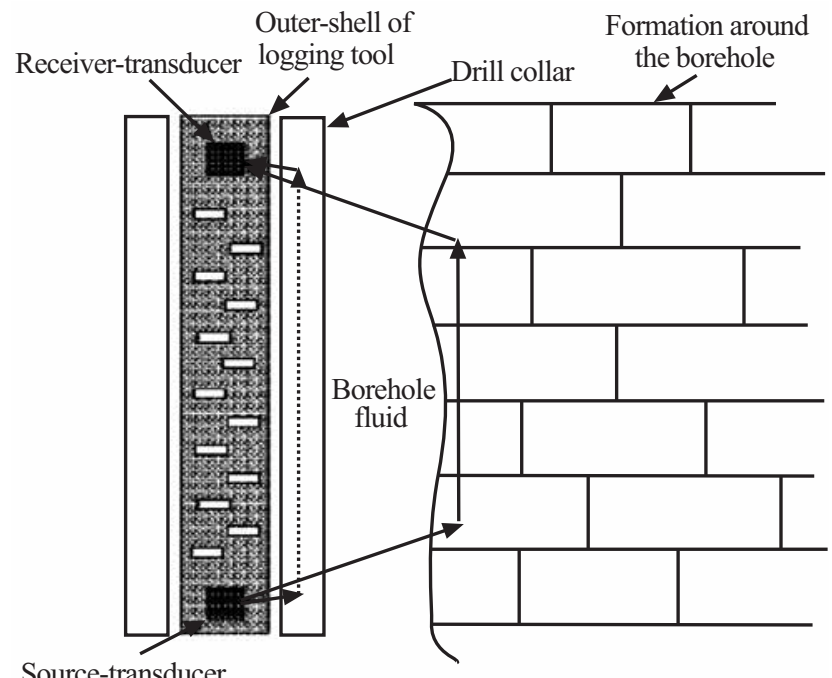

Fig. (10). A schematic plot of propagation paths of acoustic signal.

\subsection{Reflection/Refraction Between Two Anisotropy Rock Slabs}

Amplitude variations with offset (AVO) is one of the most important analyses in studies of reflection coefficient. $P$-wave amplitude variation with respect to an incidence angle is affected by acoustic impedances of $P$ - and $S V$-waves on both sides of the reflector. Ostrander [17] showed that AVO anomalies can indicate areas of Poisson's ratio change and are direct hydrocarbon indicators. However, anisotropy has potentially significant effects on AVO application and time-depth conversion of seismic data $[18,19]$. It is well known that elastic anisotropy is so common in Earth's interior that it is virtually impossible to avoid it in geophysical studies. Meanwhile, rock anisotropy is generally described as transversely isotropic and the presence of this kind of rock anisotropy can severely distort the AVO analysis.

In a boundary between two transversely isotropic media with a vertical axis of symmetry (VTI), two $4^{\text {th }}$ order polynomials have been established for calculations of reflection/refraction angles [18],

$$
\begin{aligned}
& B_{1}^{(1)} \sin ^{4} \theta^{(1,3)}+B_{3}^{(1)} \sin ^{2} \theta^{(1,3)}+B_{5}^{(1)}=0, \\
& B_{1}^{(2)} \sin ^{4} \theta^{(2,4)}+B_{3}^{(2)} \sin ^{2} \theta^{(2,4)}+B_{5}^{(2)}=0 .
\end{aligned}
$$


In case of a $P$-wave impinging on the boundary between two VTI media, the relationship between displacement and traction across boundary in terms of the reflection/refraction coefficients can be written as

$\mathbf{A R}=\overrightarrow{\mathrm{B}}$,

where, $\mathbf{A}$ is a 4 by 4 matrix, and $\vec{R}$ and $\overrightarrow{\mathrm{B}}$ are the 4-element vectors. The matrix elements of $\mathbf{A}$ are given by $a_{11}=u_{x}^{(1)}$, $a_{12}=-u_{x}^{(2)}, \quad a_{13}=-u_{z}^{(3)}, \quad a_{14}=u_{z}^{(4)}, \quad a_{21}=u_{z}^{(1)}, \quad a_{22}=u_{z}^{(2)}$, $a_{23}=-u_{x}^{(3)}$,

$a_{24}=-u_{x}^{(4)}, a_{31}=\frac{c_{13}^{(i n)} u_{x}^{(1)} \sin \theta^{(1)}+c_{33}^{(1)} u_{z}^{(1)} \cos \theta^{(1)}}{v^{(1)}\left(\theta^{(1)}\right)}$,

$a_{32}=-\frac{c_{13}^{(r e)} u_{x}^{(2)} \sin \theta^{(2)}+C_{33}^{(r e)} u_{z}^{(2)} \cos \theta^{(2)}}{v^{(2)}\left(\theta^{(2)}\right)}$,

$a_{33}=-\frac{c_{13}^{(i n)} u_{z}^{(3)} \sin \theta^{(3)}+C_{33}^{(i n)} u_{x}^{(3)} \cos \theta^{(3)}}{v^{(3)}\left(\theta^{(3)}\right)}$,

$a_{34}=-\frac{c_{33}^{(r)} u_{x}^{(4)} \sin \theta^{(4)}+c_{13}^{(r e)} u_{z}^{(4)} \cos \theta^{(4)}}{v^{(4)}\left(q^{(4)}\right)}$,

$a_{41}=\frac{c_{44}^{(i n)}\left(u_{x}^{(1)} \cos \theta^{(1)}+u_{z}^{(1)} \sin \theta^{(1)}\right)}{v^{(1)}\left(\theta^{(1)}\right)}$,

$a_{42}=\frac{c_{44}^{(r e)}\left(u_{x}^{(2)} \cos \theta_{2}+u_{z}^{(2)} \sin \theta_{2}\right)}{v^{(2)}\left(\theta^{(2)}\right)}$

$a_{43}=-\frac{c_{44}^{(i n)}\left(u_{x}^{(3)} \sin \theta^{(3)}+u_{z}^{(3)} \cos \theta^{(3)}\right)}{v^{(3)}\left(\theta^{(3)}\right)}$

$a_{44}=-\frac{c_{44}^{(r e)}\left(u_{z}^{(4)} \cos \theta^{(4)}+u_{x}^{(4)} \sin \theta^{(4)}\right)}{v^{(4)}\left(\theta^{(4)}\right)}$.

The elements of $\overrightarrow{\mathrm{B}}$ vector are given as $b_{1}=-u_{x}^{(1)}$, $b_{2}=u_{z}^{(1)}, \quad b_{3}=-\frac{c_{13}^{(i n)} u_{x}^{(1)} \sin \theta^{(1)}+c_{33}^{(i n)} u_{z}^{(1)} \cos \theta^{(1)}}{v^{(1)}\left(\theta_{1}\right)}, \quad$ and $b_{4}=\frac{c_{44}^{(i n)}\left(u_{x}^{(1)} \cos \theta^{(1)}+u_{z}^{(1)} \sin \theta^{(1)}\right)}{v^{(1)}\left(\theta^{(1)}\right)}$. In all these equations mentioned above, the superscripts $\{m\}=\{0,1,2,3,4\}$ denote incident $P$-wave or $S V$-wave $(m=0)$, the reflected $P$-wave $(m=1)$, refracted $P$-wave $(m=2)$, reflected $S V$-wave $(m=3)$, and refracted $S V$-wave $(m=4) ;\{n\}=\{i n, r e\}$ denote the incidence medium and refraction medium. For the elements in $\overrightarrow{\mathrm{R}}$ vector, $R^{(1)}$ is the reflection coefficient of quasi- $P$ to quasi- $P$ wave reflection, $R^{(2)}$ is the refraction coefficient of quasi- $P$ to quasi- $P$ wave refraction, $R^{(3)}$ is the reflection coefficient of quasi- $P$ to quasi- $S V$ wave reflection and $R^{(4)}$ is the refraction coefficient of quasi- $P$ to quasi- $S V$ wave refraction. $u_{x}^{(m)}$ and $u_{z}^{(m)}$ are the polarization coefficients of the incident and mode conversion waves, and $v^{(m)}$ is the phase velocity for the above waves. $c_{i j}^{(n)}$ is the elastic stiffness of incidence and refraction media.

We have performed calculations of the reflection/refraction coefficients based on Eqs. (5)-(7), using the anisotropic parameters of two sedimentary rocks listed in Table 4. The calculated results are plotted in Fig. (11) and are readily used for AVO analysis of seismic exploration data [20].

Table 4. Anisotropic and Physical Parameters of Rocks, where, $\alpha$ and $\beta$ are Vertical Velocity of $P$-Wave and $S V$-Wave in VTI Medium, Respectively; $\varepsilon, \delta^{*}$ and $\gamma$ are the Anisotropic Parameters of VTI Medium. A-Shale Stands for Anisotropic Shale and T-Sandstone Stands for Taylor Sandstone

\begin{tabular}{|c|c|c|c|c|c|c|}
\hline \multirow{2}{*}{ Medium } & $\alpha$ & $\beta$ & $\rho$ & \multicolumn{3}{|c|}{ Ansotropy Parameters } \\
\cline { 2 - 7 } & $(\boldsymbol{m} / \mathbf{s})$ & $(\mathbf{m} / \mathbf{s})$ & $\left(\mathrm{g} / \mathrm{cm}^{\mathbf{3}}\right)$ & $\boldsymbol{\varepsilon}$ & $\boldsymbol{\delta}^{*}$ & $\gamma$ \\
\hline \hline A-shale & 2745 & 1508 & 2.340 & 0.103 & -0.073 & 0.345 \\
\hline T-sandstone & 3368 & 1829 & 2.500 & 0.110 & -0.127 & 0.255 \\
\hline
\end{tabular}

\subsection{Technology to Inverse Oil Reservoir by Logging and Seismic Data}

For various possible geological structures, e.g. thin-out, top-lap, down-lap, etc. a seismic wavelet dictionary can be established using logging data from several oil wells in a given region. A match pursuit can be performed for words in the dictionary with the seismic exploration data obtained from this region [21, 22]. Then, the reflectivity series of underground formation can be obtained from the measured seismic wavelets and from the words in the seismic wavelet dictionary. By observing the amplitudes and phases of the obtained reflectivity series, an oil reservoir may be revealed.

In summary, this technology may be classified as two parts: creation of the seismic wavelet dictionary and software processing of reflectivity series inversion.

From the seismic wavelet dictionary, the words presented in Fig. (12) show a geological structure of a thin-out. Those in Fig. (13) present a geological structure of an inter-bed, and those in Fig. (14) reflect a geological structure with two down-laps.

In calculations of the formation reflectivity series, a software program is used to scan all words in the seismiclogging dictionary with every actual measured seismic signal wavelet. This scan would locate the maximum correlation coefficient. According to the shift invariance norm, the iterative calculations are performed by utilizing a match pursuit algorithm. When the calculation is converged, a minimal residual between the "words" with a maximal correlation coefficient is obtained. Then, the trace of actual seismic signal wavelet is reached to locate the desired oil-gas reservoirs. 


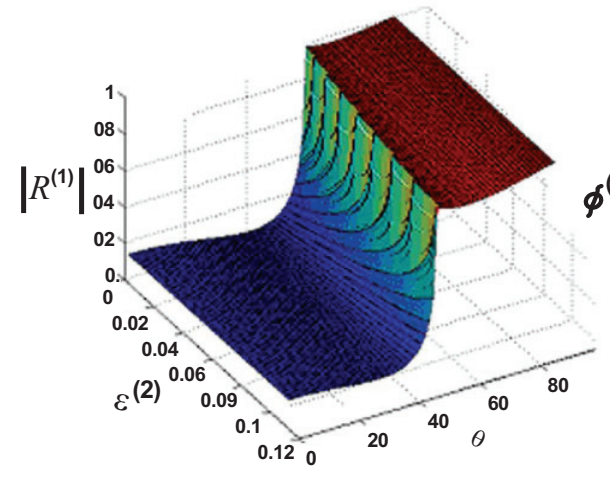

Modulus

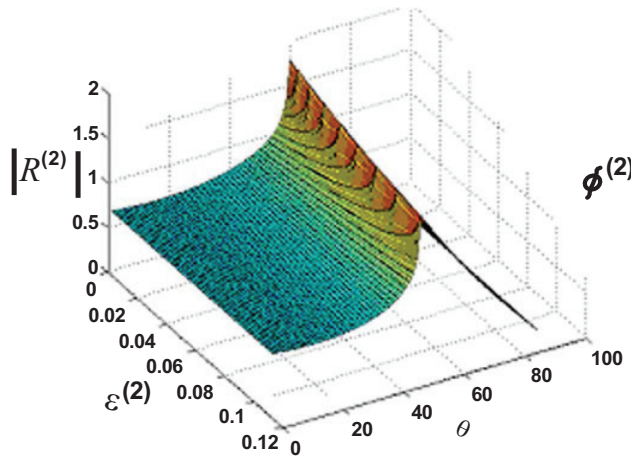

Modulus

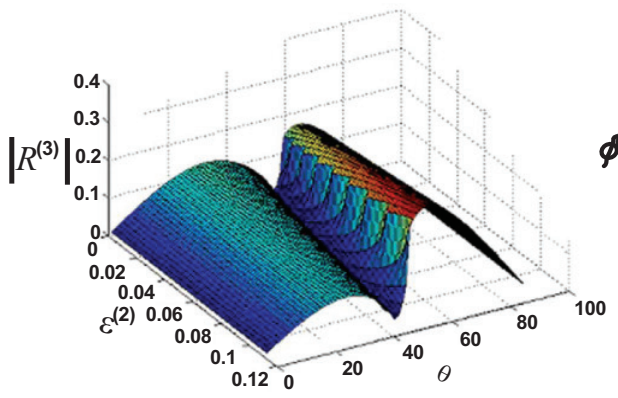

Modulus

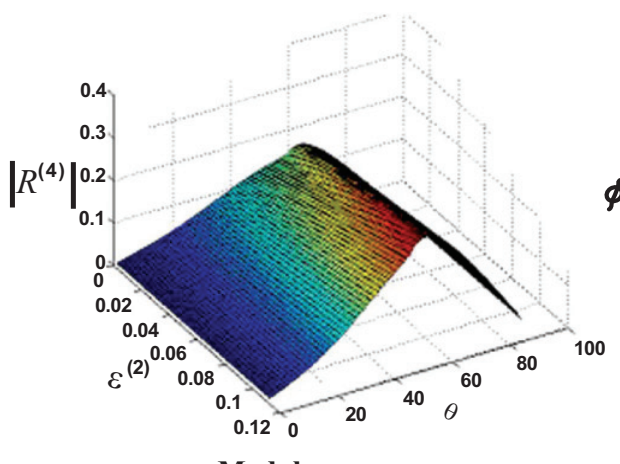

Modulus

(b)

(c) (a)

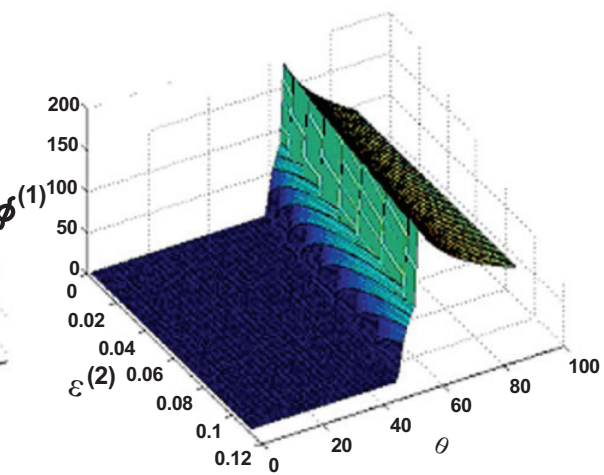

Phase shift

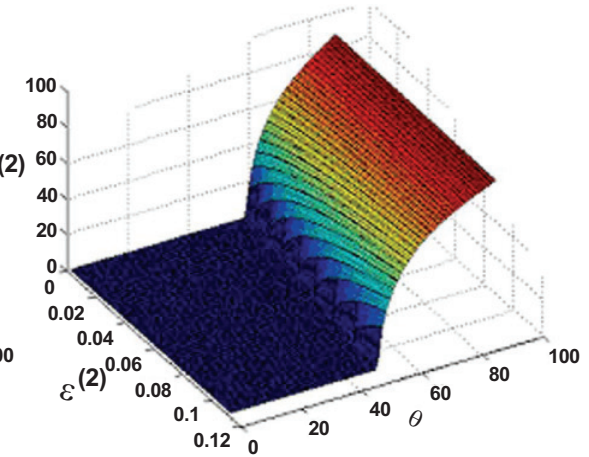

Phase shift

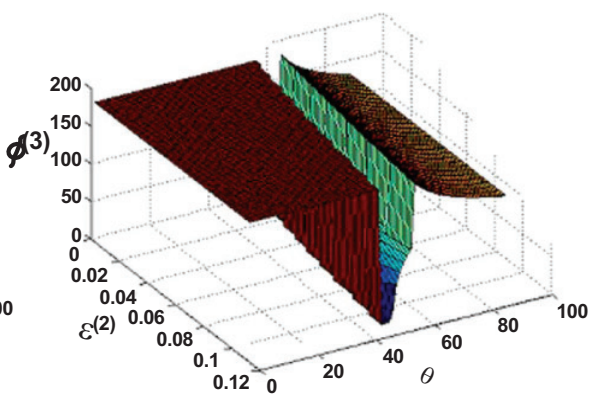

Phase shift

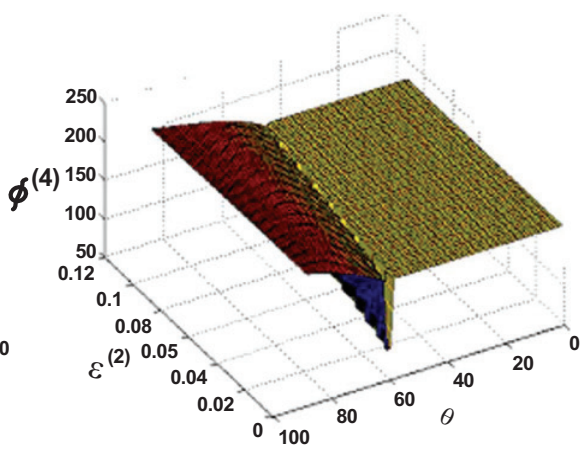

(d)

Phase shift

Fig. (11). Reflection/refraction coefficients versus $\theta$ and $\varepsilon^{(r e)}$ when $\varepsilon^{(i n)}, \delta^{*(i n)}$ and $\delta^{*(r e)}$ are fixed. In the figures, $\phi^{(1)}, \phi^{(2)}, \phi^{(3)}$ and $\phi^{(4)}$ are the phase of $R^{(1)}, R^{(2)}, R^{(3)}$ and $R^{(4)}$, respectively. 


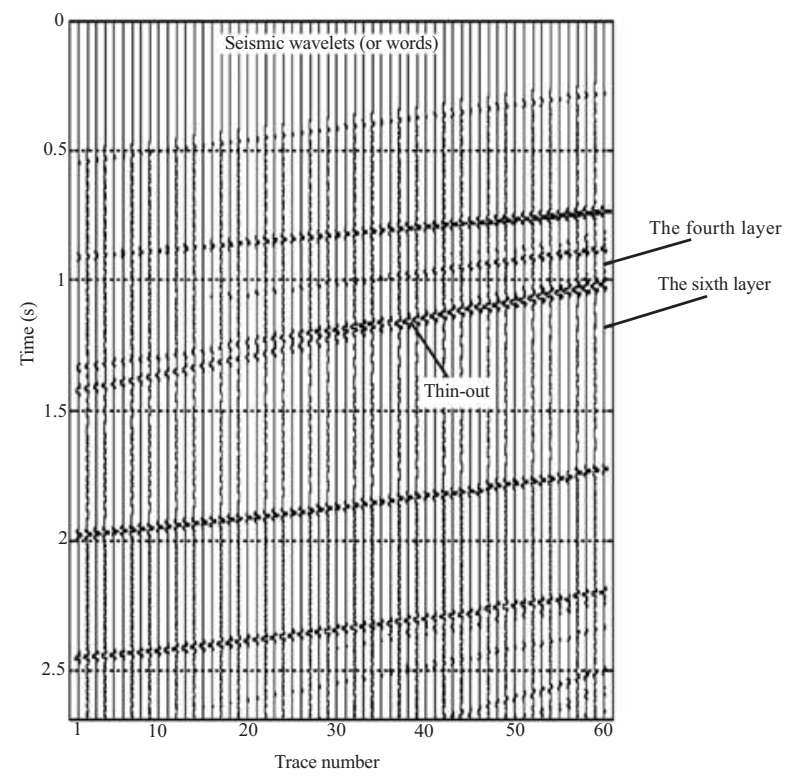

Fig. (12). A geological structure of a thin-out.

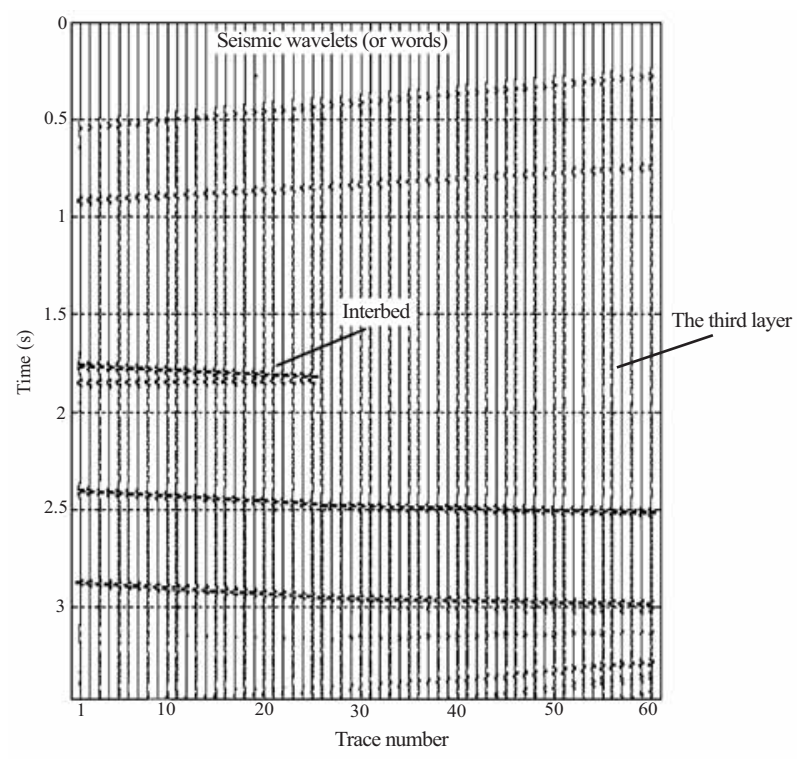

Fig. (13). A geological structure of an inter-bed.

\section{CONCLUDING REMARKS}

So far, we have discussed an improved ALTN network model for acoustical application to petroleum logging and seismic exploration. It is important to note that in studies of this new model, we identified intrinsic relationships amongst various physical quantities, such as driving-voltage signal, electric-acoustic conversion of source-transducer, acousticelectric conversion of receiver-transducer, measured logging signal, and the propagation media.

Amongst the most important physical concepts proposed in this paper are acoustic-electric analogue and a drivingvoltage convolution with electric-acoustic impulse response. The latter concept led to a practical and improved petroleum logging ALTN in which the convolution of the driving- voltage signal with the electric-acoustic impulse response function was used to substitute for some assumed acousticsource functions. These assumed functions are usually used in traditional acoustic-logging models, e.g. Tsang wavelet, Ricker wavelet, Gaussian impulse wavelet, etc.

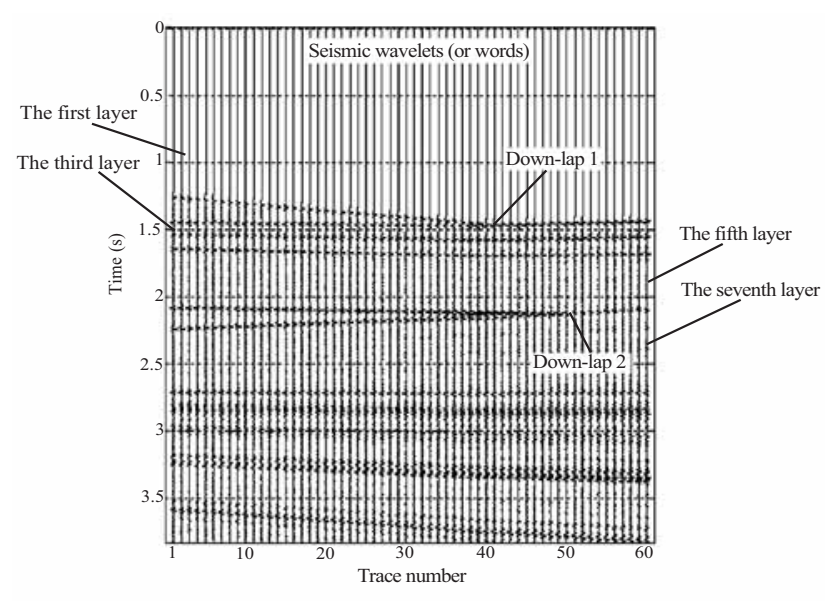

Fig. (14). A geological structure with two down-laps.

The anisotropic effects on reflection/refraction between two different rock-slabs are discussed. Correspondingly, a new fast algorithm for calculation of reflection/refraction coefficients has been presented. It provides insight into analysis of amplitude variations with offset (AVO) for exploring new oil reservoirs or gas fields accurately.

Getting into the prediction of new oil reservoirs and/or gas fields, the new algorithm leads to the construction of a seismic wavelet-dictionary which can be constructed by logging data from several oil wells in a given region. The reflectivity series of the formation can be obtained accurately by using the match-pursuit algorithm, the seismic wavelet dictionary and the measured seismic reflection data.

Within the framework of the newly proposed network model, there are several other important issues discussed besides the discussions above, including (i) the notation of addition and multiplication of acoustic-logging ALTN transmission network, (ii) the technology of eliminating acoustic signal propagation in the drill-collar for acousticlogging while drilling, and (iii) the effect of rock anisotropy on reflection/refraction coefficients and its relation to an accurate AVO analysis.

In summary, the newly proposed ALTN network model describes the acoustic-logging process based strictly on a physical mechanism. It is more practical and much closer to the actual situation of acoustic logging than any earlier acoustic-logging models. Applications of the new network model in analysis of acoustic-logging process lead to accurate acoustic-logging information, such as propagation velocity, signal amplitude, wavelet phase, and frequency spectrum of acoustic-logging signal in the formation.

\section{CONFLICT OF INTEREST}

The authors confirm that this article content has no conflicts of interest. 


\section{ACKNOWLEDGEMENTS}

This work is supported in part by a grant (No. 40974078) from the National Natural Science Foundation of China and by the Physical Sciences Division at The University of Chicago.

\section{REFERENCES}

[1] Larose E, Rosny J, Margerin L, et al. Observation of multiple scattering of $\mathrm{kHz}$ vibrations in a concrete structure and application to monitoring weak changes. Physical Rev E Stat Nonlin Soft Matter Phys 2006; 73(1): 016609.

[2] Tsang L, Rader D. Numerical evaluation of the transient acoustic waveform due to a point source in a fluid-filled bore hole. Geophysics 1979; 44: 1706-20.

[3] Gibson Jr RL, Peng C. Low- and high-frequency radiation from seismic sources in cased boreholes. Geophysics 1994; 59: 1780-5.

[4] Cheng $\mathrm{CH}$, Toksöz MN. Elastic wave propagation in a fluidfilled borehole and synthetic acoustic logs. Geophysics 1981; 46: 104253.

[5] Cerveny V. Seismic ray theory. Cambridge: Cambridge University Press 2001.

[6] Thomsen L. Weak elastic anisotropy. Geophysics 1986; 51: 195466.

[7] Wang Y. Seismic time-frequency spectral decomposition by matching pursuit. Geophysics 2007; 72: 13-20.

[8] Fa L, Castagna JP, Hovem JM. Derivation and simulation of source function for acoustic-logging. IEEE Ultrasonics Symposium Proceedings; 1999 November; Lake Tahoe, USA 1999.

[9] Fa L, Castagna JP, Hovem JM, Dong DQ. An acoustic-logging transmission-network model. J Acoust Soc Am 2002; 111: 215865 .
[10] Fa L, Castagna JP, Suarez-Rivera R, Sun P. An acoustic-logging transmission-network model (continued): Addition and multiplication ALTNs. J Acoust Soc Am 2003; 113: 2698-703.

[11] The Product Catalogue of CHANNEL Industries, Inc., 839 Ward Drive, Santa Barbara, CA 93111, USA 1992

[12] Gong W. Full waveform analysis of acoustic well logging and its multidimimensional procession methods. Ph.D. thesis. China: Southeast University, July 1988.

[13] Fa L, Castagna JP, Hovem JM, Dong D. An acoustic-logging transmission-network model. J Acoust Soc Am 2002; 111: 215865.

[14] Fa L, Xie WY, Tian Y, Zhao MS, MA L, DONG DQ. Effects of electric-acoustic and acoustic-electric conversions of transducers on acoustic-logging signal. Chin Sci Bull 2012; 57: 1246-60.

[15] Fa L. Application of phase control array technique to sonic logging. Proceedings of The China-Japan Joint Conference on Ultrasonics; 1987 April, Nanjing: China 1987.

[16] Fa L, Ma HF. Design of a new type of array transmitting sonic logging. Acta Petrolei Sin 1991; 12: 52-7.

[17] Ostrander WJ. Plane wave reflection coefficients for gas sands at nonnormal angles of incidence. Geophysics 1984; 49: 1637-48.

[18] Fa L, Brown RL, Castagna JP. Anomalous post-critical refraction behavior for certain transversely isotropic media. J Acoust Soc Am 2006; 120: 3479-92.

[19] Zhao Y, Zhao N, Fa L, Zhao M. Seismic signal and data analysis of rock media with vertical anisotropy. J Mod Phys 2013; 4: 11-8.

[20] Castagna JP. Offset-dependent reflectivity: theory and practice of AVO analysis. Oklahama, Tuba, USA: Society of Exploration Geophysicists 1993.

[21] Mallat S, Zhang Z. Matching pursuit with time-frequency dictionaries. IEEE Trans Signal Proc 1993; 44(12): 3397-415.

[22] Davis G, Mallat S, Avellaneda M. Adaptive greedy approximations. Constr Approximation 1997; 13: 57-98. 